University of Texas at El Paso

ScholarWorks@UTEP

$12-2014$

\title{
How Much For an Interval? a Set? a Twin Set? a p-Box? A Kaucher Interval? Towards an Economics-Motivated Approach to Decision Making Under Uncertainty
}

Joe Lorkowski

The University of Texas at El Paso, lorkowski@computer.org

Vladik Kreinovich

The University of Texas at El Paso, vladik@utep.edu

Follow this and additional works at: https://scholarworks.utep.edu/cs_techrep

Part of the Computer Engineering Commons

Comments:

Technical Report: UTEP-CS-14-78

\section{Recommended Citation}

Lorkowski, Joe and Kreinovich, Vladik, "How Much For an Interval? a Set? a Twin Set? a p-Box? A Kaucher Interval? Towards an Economics-Motivated Approach to Decision Making Under Uncertainty" (2014). Departmental Technical Reports (CS). 871.

https://scholarworks.utep.edu/cs_techrep/871

This Article is brought to you for free and open access by the Computer Science at ScholarWorks@UTEP. It has been accepted for inclusion in Departmental Technical Reports (CS) by an authorized administrator of ScholarWorks@UTEP.For more information, please contact Iweber@utep.edu. 


\title{
How Much For an Interval? a Set? a Twin Set? a p-Box? A Kaucher Interval? Towards an Economics-Motivated Approach to Decision Making Under Uncertainty \\ Joe Lorkowski and Vladik Kreinovich University of Texas at El Paso, El Paso, TX 79968, USA lorkowski@computer.org, vladik@utep.edu
}

\begin{abstract}
A natural idea of decision making under uncertainty is to assign a fair price to different alternatives, and then to use these fair prices to select the best alternative. In this paper, we show how to assign a fair price under different types of uncertainty.
\end{abstract}

\section{Decision Making under Uncertainty: Formu- lation of the Problem}

Need for decision making. In many practical situations, we have several alternatives, and we need to select one of these alternatives. For example:

- a person saving for retirement needs to find the best way to invest money;

- a company needs to select a location for its new plant;

- a designer must select one of several possible designs for a new airplane;

- a medical doctor needs to select a treatment for a patient, etc.

Need for decision making under uncertainty. Decision making is the easiest if we know the exact consequences of selecting each alternative. Often, however, we only have an incomplete information about consequences of different alternative, and we need to select an alternative under this uncertainty.

How decisions under uncertainty are made now. Traditional decision theory (see, e.g., $[8,12]$ ) assumes that for each alternative $a$, we know the probability $p_{i}(a)$ of different outcomes $i$. It can be proven that preferences of a 
rational decision maker can be described by utilities $u_{i}$ so that an alternative $a$ is better if its expected utility $u(a) \stackrel{\text { def }}{=} \sum_{i} p_{i}(a) \cdot u_{i}$ is larger.

Problem. Often, we do not know the probabilities $p_{i}(a)$. How can we then make a decision? This is the problem that we will deal with in this paper - by explaining how to assign a fair price to each alternatives, so that we can select between several alternatives by comparing their fair prices.

\section{Fair Price Approach: Main Idea and Its Ap- plication to Interval Uncertainty}

Fair price approach: an idea. When we have a full information about an object, then we can express our desirability of each possible situation by declaring a price that we are willing to pay to get involved in this situation. Once these prices are set, we simply select the alternative for which the participation price is the highest.

In decision making under uncertainty, it is not easy to come up with a fair price. A natural idea is therefore to develop techniques for producing such fair prices. These prices can then be used in decision making, to select an appropriate alternative.

Case of interval uncertainty. In the ideal case, we know the exact gain $u$ of selecting an alternative. A more realistic case is when we only know the lower bound $u$ and the upper bound $\bar{u}$ on this gain - and we do not know which values $u \in[\underline{u}, \bar{u}]$ are more probable probable or less probable. This situation is known as interval uncertainty.

Interval uncertainty: how decisions are made now. A widely used idea was proposed in the 1950s by a future Nobelist L. Hurwicz $[5,8]$ : we should select an alternative that maximizes the value $\alpha_{H} \cdot \bar{u}(a)+\left(1-\alpha_{H}\right) \cdot \underline{u}(a)$. Here, the parameter $\alpha_{H} \in[0,1]$ described the optimism level of a decision maker:

- $\alpha_{H}=1$ means optimism;

- $\alpha_{H}=0$ means pessimism;

- $0<\alpha_{H}<1$ combines optimism and pessimism.

Fair price approach to decision making under interval uncertainty: general description. We want to assign, to each interval $[\underline{u}, \bar{u}]$, a number $P([\underline{u}, \bar{u}])$ describing the fair price of this interval.

Let us list the natural properties of the fair price.

Fair price should be within the given interval. Since we know that the utility $u$ is smaller than or equal to $\bar{u}$, the corresponding fair price should also smaller than or equal to this bound: $P([\underline{u}, \bar{u}]) \leq \bar{u}$. Similarly, since we know that $\underline{u} \leq u$, we should have $\underline{u} \leq P([\underline{u}, \bar{u}])$. 
Thus, the fair price of an interval should be located in this interval:

$$
P([\underline{u}, \bar{u}]) \in[\underline{u}, \bar{u}] .
$$

Monotonicity. Suppose that we keep the lower endpoint $\underline{u}$ intact but increase the upper bound. This means that we keep all the previous possibilities, but we also add new possibilities, with a higher gain. In this case, it is reasonable to require that after this addition, the fair price should either increase or remain the same, but it should definitely not decrease:

$$
\text { if } \underline{u}=\underline{v} \text { and } \bar{u}<\bar{v} \text { then } P([\underline{u}, \bar{u}]) \leq P([\underline{v}, \bar{v}]) \text {. }
$$

Similarly, if we dismiss some low-gain alternatives, this should increase (or at least not decrease) the fair price:

$$
\text { if } \underline{u}<\underline{v} \text { and } \bar{u}=\bar{v} \text { then } P([\underline{u}, \bar{u}]) \leq P([\underline{v}, \bar{v}]) .
$$

Additivity. Let us consider the situation when we have two consequent independent decisions. In this case, we can either consider two decision processes separately, or we can consider a single decision process in which we select a pair of alternatives:

- the 1st alternative corresponding to the 1st decision, and

- the 2 nd alternative corresponding to the 2 nd decision.

If we are willing to pay the amount $u$ to participate in the first process, and we are willing to pay the amount $v$ to participate in the second decision process, then it is reasonable to require that we should be willing to pay $u+v$ to participate in both decision processes.

Additivity: case of interval uncertainty. In the case of interval uncertainty, about the gain $u$ from the first alternative, we only know that this (unknown) gain is in $[\underline{u}, \bar{u}]$. Similarly, about the gain $v$ from the second alternative, we only know that this gain belongs to the interval $[\underline{v}, \bar{v}]$.

The overall gain $u+v$ can thus take any value from the interval

$$
[\underline{u}, \bar{u}]+[\underline{v}, \bar{v}] \stackrel{\text { def }}{=}\{u+v: u \in[\underline{u}, \bar{u}], v \in[\underline{v}, \bar{v}]\} .
$$

It is easy to check that (see, e.g., $[6,10])$ :

$$
[\underline{u}, \bar{u}]+[\underline{v}, \bar{v}]=[\underline{u}+\underline{v}, \bar{u}+\bar{v}] .
$$

Thus, for the case of interval uncertainty, the additivity requirement about the fair prices takes the form

$$
P([\underline{u}+\underline{v}, \bar{u}+\bar{v}])=P([\underline{u}, \bar{u}])+P([\underline{v}, \bar{v}]) .
$$


So, we arrive at the following definition:

Definition 1. By a fair price under interval uncertainty, we mean a function $P([\underline{u}, \bar{u}])$ for which:

- $\underline{u} \leq P([\underline{u}, \bar{u}]) \leq \bar{u}$ for all $\underline{u}$ and $\bar{u}$ (conservativeness);

- if $\underline{u}=\underline{v}$ and $\bar{u}<\bar{v}$, then $P([\underline{u}, \bar{u}]) \leq P([\underline{v}, \bar{v}])$ (monotonicity);

- (additivity) for all $\underline{u}, \bar{u}, \underline{v}$, and $\bar{v}$, we have

$$
P([\underline{u}+\underline{v}, \bar{u}+\bar{v}])=P([\underline{u}, \bar{u}])+P([\underline{v}, \bar{v}]) .
$$

Proposition 1. [9] Each fair price under interval uncertainty has the form

$$
P([\underline{u}, \bar{u}])=\alpha_{H} \cdot \bar{u}+\left(1-\alpha_{H}\right) \cdot \underline{u} \text { for some } \alpha_{H} \in[0,1] .
$$

Comment. We thus get a new justification of Hurwicz optimism-pessimism criterion.

\section{Proof.}

$1^{\circ}$. Due to monotonicity, $P([u, u])=u$.

$2^{\circ}$. Also, due to monotonicity, $\alpha_{H} \stackrel{\text { def }}{=} P([0,1]) \in[0,1]$.

$3^{\circ}$. For $[0,1]=[0,1 / n]+\ldots+[0,1 / n](n$ times $)$, additivity implies $\alpha_{H}=$ $n \cdot P([0,1 / n])$, so $P([0,1 / n])=\alpha_{H} \cdot(1 / n)$.

$4^{\circ}$. For $[0, m / n]=[0,1 / n]+\ldots+[0,1 / n]$ ( $m$ times $)$, additivity implies

$$
P([0, m / n])=\alpha_{H} \cdot(m / n) .
$$

$5^{\circ}$. For each real number $r$, for each $n$, there is an $m$ such that $m / n \leq r \leq$ $(m+1) / n$. Monotonicity implies

$$
\alpha_{H} \cdot(m / n)=P([0, m / n]) \leq P([0, r]) \leq P([0,(m+1) / n])=\alpha_{H} \cdot((m+1) / n) .
$$

When $n \rightarrow \infty, \alpha_{H} \cdot(m / n) \rightarrow \alpha_{H} \cdot r$ and $\alpha_{H} \cdot((m+1) / n) \rightarrow \alpha_{H} \cdot r$, hence $P([0, r])=\alpha_{H} \cdot r$.

$6^{\circ}$. For $[\underline{u}, \bar{u}]=[\underline{u}, \underline{u}]+[0, \bar{u}-\underline{u}]$, additivity implies $P([\underline{u}, \bar{u}])=\underline{u}+\alpha_{H} \cdot(\bar{u}-\underline{u})$. The proposition is proven. 


\section{Case of Set-Valued Uncertainty}

Formulation of the problem. In some cases, in addition to knowing that the actual gain belongs to the interval $[\underline{u}, \bar{u}]$, we also know that some values from this interval cannot be possible values of this gain.

For example, if we buy an obscure lottery ticket for a simple prize-or-noprize lottery from a remote country, we either get the prize or lose the money. In this case, the set of possible values of the gain consists of two values.

In general, instead of a (bounded) interval of possible values, we can consider a general bounded set of possible values. So, what is the fair price under such set-valued uncertainty?

Definition 2. By a fair price under set-valued uncertainty, we mean a function $P$ that assigns, to every bounded closed set $S$, a real number $P(S)$, for which:

- $P([\underline{u}, \bar{u}])=\alpha_{H} \cdot \bar{u}+\left(1-\alpha_{H}\right) \cdot \underline{u}$ (conservativeness);

- $P\left(S+S^{\prime}\right)=P(S)+P\left(S^{\prime}\right)$, where $S+S^{\prime} \stackrel{\text { def }}{=}\left\{s+s^{\prime}: s \in S, s^{\prime} \in S^{\prime}\right\}$ (additivity).

Proposition 2. Each fair price under set uncertainty has the form $P(S)=$ $\alpha_{H} \cdot \sup S+\left(1-\alpha_{H}\right) \cdot \inf S$.

Proof. It is easy to check that each bounded closed set $S$ contains its infimum $\underline{s} \stackrel{\text { def }}{=} \inf S$ and supremum $\underline{s} \stackrel{\text { def }}{=} \sup S:\{\underline{s}, \bar{s}\} \subseteq S \subseteq[\underline{s}, \bar{s}]$. Thus,

$$
[2 \underline{s}, 2 \bar{s}]=\{\underline{s}, \bar{s}\}+[\underline{s}, \bar{s}] \subseteq S+[\underline{s}, \bar{s}] \subseteq[\underline{s}, \bar{s}]+[\underline{s}, \bar{s}]=[2 \underline{s}, 2 \bar{s}] .
$$

So, $S+[\underline{s}, \bar{s}]=[2 \underline{s}, 2 \bar{s}]$. By additivity, we conclude that $P(S)+P([\underline{s}, \bar{s}])=$ $P([2 \underline{s}, 2 \bar{s}])$. Due to conservativeness, we know the fair prices $P([\underline{s}, \bar{s}])$ and $P([2 \underline{s}, 2 \bar{s}])$. Thus, we can conclude that

$P(S)=P([2 \underline{s}, 2 \bar{s}])-P([\underline{s}, \bar{s}])=\left(\alpha_{H} \cdot(2 \bar{s})+\left(1-\alpha_{H}\right) \cdot(2 \underline{s})\right)-\left(\alpha_{H} \cdot \bar{s}+\left(1-\alpha_{H}\right) \cdot \underline{s}\right)$, hence indeed $P(S)=\alpha_{H} \cdot \bar{s}+\left(1-\alpha_{H}\right) \cdot \underline{s}$. The proposition is proven.

\section{Case of Probabilistic Uncertainty}

Formulation of the problem. Suppose that for some financial instrument, we know the corresponding probability distribution $\rho(x)$ on the set of possible gains $x$. What is the fair price $P$ for this instrument?

Analysis of the problem. Due to additivity, the fair price for $n$ copies of this instrument is $n \cdot P$. According to the Large Numbers Theorem, for large $n$, the average gain tends to the mean value $\mu=\int x \cdot \rho(x) d x$.

Thus, the fair price for $n$ copies of the instrument is close to $n \cdot \mu: n \cdot P \approx n \cdot \mu$. The larger $n$, the closer the averages. So, in the limit, we get $P=\mu$.

Conclusion. The fair price under probabilistic uncertainty is equal to the average gain $\mu=\int x \cdot \rho(x) d x$. 


\section{Case of p-Box Uncertainty}

Formulation of the problem. Probabilistic uncertainty means that for every $x$, we know the value of the $\operatorname{cdf} F(x) \stackrel{\text { def }}{=} \operatorname{Prob}(\eta \leq x)$. In practice, we often only have partial information about these values. In this case, for each $x$, we only know an interval $[\underline{F}(x), \bar{F}(x)]$ containing the actual (unknown) value $F(x)$.

The interval-valued function $[\underline{F}(x), \bar{F}(x)]$ is known as a $p$-box $[2,3]$. What is the fair price of a p-box?

Analysis of the problem. The only information that we have about the cdf is that $F(x) \in[\underline{F}(x), \bar{F}(x)]$. For each possible $F(x)$, for large $n, n$ copies of the instrument are $\approx$ equivalent to $n \cdot \mu$, where $\mu=\int x d F(x)$.

For different $F(x)$, values of $\mu$ for an interval $[\mu, \bar{\mu}]$, where $\mu=\int x d \bar{F}(x)$ and $\bar{\mu}=\int x d \underline{F}(x)$. Thus, the price of a p-box is equal to the price of an interval $[\mu, \bar{\mu}]$.

We already know that the fair price of this interval is equal to

$$
\alpha_{H} \cdot \bar{\mu}+\left(1-\alpha_{H}\right) \cdot \underline{\mu} .
$$

Thus, we arrive at the following conclusion.

Conclusion. The fair price of a p-box $[\underline{F}(x), \bar{F}(x)]$ is $\alpha_{H} \cdot \bar{\mu}+\left(1-\alpha_{H}\right) \cdot \underline{\mu}$, where $\underline{\mu}=\int x d \bar{F}(x)$ and $\bar{\mu}=\int x d \underline{F}(x)$.

\section{Case of Kaucher (Improper) Intervals}

Formulation of the problem. What is the price for an improper interval $[\underline{x}, \bar{x}]$, with $\underline{x}>\bar{x}$ (see, e.g., $[7,13])$ ?

Analysis of the problem. Let us use additivity. Here,

$$
[\underline{x}, \bar{x}]+[\bar{x}, \underline{x}]=[\underline{x}+\bar{x}, \underline{x}+\bar{x}] .
$$

Thus,

$$
P([\underline{x}, \bar{x}])+P([\bar{x}, \underline{x}])=P([\underline{x}+\bar{x}, \underline{x}+\bar{x}]) .
$$

We know that $P([\bar{x}, \underline{x}])=\alpha_{H} \cdot \underline{x}+\left(1-\alpha_{H}\right) \cdot \bar{x}$ and $P(\underline{x}+\bar{x})=\underline{x}+\bar{x}$. Hence:

$$
P([\underline{x}, \bar{x}])=(\underline{x}+\bar{x})-\left(\alpha_{H} \cdot \underline{x}+\left(1-\alpha_{H}\right) \cdot \bar{x}\right) .
$$

Therefore, we arrive at the following conclusion.

Conclusion. The fair price $P([\underline{x}, \bar{x}])$ of an improper interval $[\underline{x}, \bar{x}]$, with $\underline{x}>\bar{x}$, is equal to $P([\underline{x}, \bar{x}])=\alpha_{H} \cdot \bar{x}+\left(1-\alpha_{H}\right) \cdot \underline{x}$. 


\section{Case of Triples}

Formulation of the problem. Sometimes, in addition to an interval $[\underline{x}, \bar{x}]$, we also have a "most probable" value $x$ within this interval. For such triples (see, e.g., [1] and references therein), addition is defined component-wise:

$$
([\underline{x}, \bar{x}], x)+([\underline{y}, \bar{y}], y)=([\underline{x}+\underline{y}, \bar{x}+\bar{y}], x+y) .
$$

What is the fair price of such a triple?

Analysis of the problem. For triples, the additivity requirement about the fair prices takes the form

$$
P([\underline{x}+\underline{y}, \bar{x}+\bar{y}], x+y)=P([\underline{x}, \bar{x}], x)+P([\underline{y}, \bar{y}], y) .
$$

Definition 3. By a fair price under triple uncertainty, we mean a function $P([\underline{u}, \bar{u}], u)$ for which:

- $\underline{u} \leq P([\underline{u}, \bar{u}], u) \leq \bar{u}$ for all $\underline{u} \leq u \leq \bar{u}$ (conservativeness);

- if $\underline{u} \leq \underline{v}, u \leq v$, and $\bar{u} \leq \bar{v}$, then $P([\underline{u}, \bar{u}], u) \leq P([\underline{v}, \bar{v}], v)$ (monotonicity);

- (additivity) for all $\underline{u}, \bar{u}, u \underline{v}, \bar{v}$, and $v$, we have

$$
P([\underline{u}+\underline{v}, \bar{u}+\bar{v}], u+v)=P([\underline{u}, \bar{u}], u)+P([\underline{v}, \bar{v}], v) .
$$

Proposition 3. Each fair price under triple uncertainty has the form

$$
P([\underline{u}, \bar{u}], u)=\alpha_{L} \cdot \underline{u}+\left(1-\alpha_{L}-\alpha_{U}\right) \cdot u+\alpha_{U} \cdot \bar{u}, \text { where } \alpha_{L}, \alpha_{U} \in[0,1] .
$$

Proof. In general, we have

$$
([\underline{u}, \bar{u}], u)=([u, u], u)+([0, \underline{u}-u], 0)+([\underline{u}-u, 0], 0) .
$$

So, due to additivity:

$$
P([\underline{u}, \bar{u}], u)=P([u, u], u)+P([0, \underline{u}-u], 0)+P([\underline{u}-u, 0], 0) .
$$

Due to conservativeness, $P([u, u], u)=u$.

Similarly to the interval case, we can prove that $P([0, r], 0)=\alpha_{U} \cdot r$ for some $\alpha_{U} \in[0,1]$, and that $P([r, 0], 0)=\alpha_{L} \cdot r$ for some $\alpha_{L} \in[0,1]$. Thus,

$$
P([\underline{u}, \bar{u}], u)=\alpha_{L} \cdot \underline{u}+\left(1-\alpha_{L}-\alpha_{U}\right) \cdot u+\alpha_{U} \cdot \bar{u} .
$$

The proposition is proven. 


\section{Case of Twin Intervals}

Formulation of the problem. Sometimes, instead of a "most probable" value $x$, we have a "most probable" subinterval $[\underline{m}, \bar{m}] \subseteq[\underline{x}, \bar{x}]$. The resulting pair of intervals is known as a "twin interval" (see, e.g., $[4,11])$.

Analysis of the problem. For such twin intervals, addition is defined component-wise:

$$
([\underline{x}, \bar{x}],[\underline{m}, \bar{m}])+([\underline{y}, \bar{y}],[\underline{n}, \bar{n}])=([\underline{x}+\underline{y}, \bar{x}+\bar{y}],[\underline{m}+\underline{n}, \bar{m}+\bar{n}]) .
$$

Thus, the additivity requirement about the fair prices takes the form

$$
P([\underline{x}+\underline{y}, \bar{x}+\bar{y}],[\underline{m}+\underline{n}, \bar{m}+\bar{n}])=P([\underline{x}, \bar{x}],[\underline{m}, \bar{m}])+P([\underline{y}, \bar{y}],[\underline{n}, \bar{n}]) .
$$

Definition 4. By a fair price under twin uncertainty, we mean a function $P([\underline{u}, \bar{u}],[\underline{m}, \bar{m}])$ for which:

- $\underline{u} \leq P([\underline{u}, \bar{u}],[\underline{m}, \bar{m}]) \leq \bar{u}$ for all $\underline{u} \leq \underline{m} \leq \bar{m} \leq \bar{u}$ (conservativeness);

- if $\underline{u} \leq \underline{v}, \underline{m} \leq \underline{n}, \bar{m} \leq \bar{n}$, and $\bar{u} \leq \bar{v}$, then $P([\underline{u}, \bar{u}],[\underline{m}, \bar{m}]) \leq$ $P([\underline{v}, \bar{v}],[\underline{n}, \bar{n}])$ (monotonicity);

- for all $\underline{u} \leq \underline{m} \leq \bar{m} \leq \bar{u}$ and $\underline{v} \leq \underline{n} \leq \bar{n} \leq \bar{v}$, we have additivity:

$$
P([\underline{u}+\underline{v}, \bar{u}+\bar{v}],[\underline{m}+\underline{n}, \bar{m}+\bar{m}])=P([\underline{u}, \bar{u}],[\underline{m}, \bar{m}])+P([\underline{v}, \bar{v}],[\underline{n}, \bar{n}]) .
$$

Proposition 4. Each fair price under twin uncertainty has the following form, for some $\alpha_{L}, \alpha_{u}, \alpha_{U} \in[0,1]$ :

$$
P([\underline{u}, \bar{u}],[\underline{m}, \bar{m}])=\underline{m}+\alpha_{u} \cdot(\bar{m}-\underline{m})+\alpha_{U} \cdot(\bar{U}-\bar{m})+\alpha_{L} \cdot(\underline{u}-\underline{m}) .
$$

Proof. In general, we have

$$
\begin{gathered}
([\underline{u}, \bar{u}],[\underline{m}, \bar{m}])=([\underline{m}, \underline{m}],[\underline{m}, \underline{m}])+([0, \bar{m}-\underline{m}],[0, \bar{m}-\underline{m}])+ \\
([0, \bar{u}-\bar{m}],[0,0])+([\underline{u}-\underline{m}, 0],[0,0)] .
\end{gathered}
$$

So, due to additivity:

$$
\begin{gathered}
P([\underline{u}, \bar{u}],[\underline{m}, \bar{m}])=P([\underline{m}, \underline{m}],[\underline{m}, \underline{m}])+P([0, \bar{m}-\underline{m}],[0, \bar{m}-\underline{m}])+ \\
P([0, \bar{u}-\bar{m}],[0,0])+P([\underline{u}-\underline{m}, 0],[0,0)] .
\end{gathered}
$$

Due to conservativeness, $P([\underline{m}, \underline{m}],[\underline{m}, \underline{m}])=\underline{m}$. Similarly to the interval case, we can prove that:

- $P([0, r],[0, r])=\alpha_{u} \cdot r$ for some $\alpha_{u} \in[0,1]$, 
- $P([0, r],[0,0])=\alpha_{U} \cdot r$ for some $\alpha_{U} \in[0,1]$;

- $P([r, 0],[0,0])=\alpha_{L} \cdot r$ for some $\alpha_{L} \in[0,1]$.

Thus,

$$
P([\underline{u}, \bar{u}],[\underline{m}, \bar{m}])=\underline{m}+\alpha_{u} \cdot(\bar{m}-\underline{m})+\alpha_{U} \cdot(\bar{U}-\bar{m})+\alpha_{L} \cdot(\underline{u}-\underline{m}) .
$$

The proposition is proven.

\section{Acknowledgments}

This work was supported in part by the National Science Foundation grants HRD-0734825 and HRD-1242122 (Cyber-ShARE Center of Excellence) and DUE-0926721, by Grant 1 T36 GM078000-01 and 1R43TR000173-01 from the National Institutes of Health, and by grant N62909-12-1-7039 from the Office of Naval Research.

The authors are thankful to all the participants of the 16th International Symposium on Scientific Computing, Computer Arithmetic, and Validated Numerics SCAN'2014 (Würzburg, German, September 21-26, 2014).

\section{References}

[1] A. J. Cole and R. Morrison, "Triplex: a system for interval arithmetic", Software: Practice and Experience, 1982, Vol. 12, No. 4, pp. 341-350.

[2] S. Ferson, Risk Assessment with Uncertainty Numbers: RiskCalc, CRC Press, Boca Raton, Florida, 2002.

[3] S. Ferson, V. Kreinovich, W. Oberkampf, and L. Ginzburg, Experimental Uncertainty Estimation and Statistics for Data Having Interval Uncertainty, Sandia National Laboratories, Report SAND2007-0939, May 2007.

[4] E. Gardefies, A. Trepat, and J. M. Janer, "SIGLA-PL/1: development and applications", In: K. L. E. Nickel (ed.), Interval Mathematics 1980, Academic Press, New York, 1980, pp. 301-315.

[5] L. Hurwicz, Optimality criteria for decision making under ignorance, Cowles Commission Discussion Paper, Statistics, 1951, No. 370.

[6] L. Jaulin, M. Kieffer, O. Didrit, and E. Walter, Applied Interval Analysis, with Examples in Parameter and State Estimation, Robust Control and Robotics, Springer-Verlag, London, 2001.

[7] E. Kaucher, "Über Eigenschaften und Anwendungsmöglichkeiten der erweiterten lntervallrechnung und des hyperbolische Fastköpers über $R$ ", Computing, 1977, Supplement 1, pp. 81-94. 
[8] R. D. Luce and R. Raiffa, Games and Decisions: Introduction and Critical Survey, Dover, New York, 1989.

[9] J. McKee, J. Lorkowski, and T. Ngamsantivong, "Note on fair price under interval uncertainty", Journal of Uncertain Systems, 2014, Vol. 8, No. 3, pp. 186-189.

[10] R. E. Moore, R. B. Kearfott, and M. J. Cloud, Introduction to Interval Analysis, SIAM Press, Philadelphia, Pennsylviania, 2009.

[11] V. M. Nesterov, "Interval and twin arithmetics", Reliable Computing, 1997, Vol. 3, No. 4, pp. 369-380.

[12] H. Raiffa, Decision Analysis: Introductory Lectures on Choices Under Uncertainty, Mcgraw-Hill, 1997.

[13] M. A. Sainz, J. Armengol, R. Calm, P. Herrero, L. Jorba and J. Vehi, Modal Interval Analysis, Springer, Berlin, Heidelberg, New York, 2014. 